[1] S. Gorfman. Cryst Rev, 20(3): 210-232, (2014).

[2] S. Gorfman, H. Choe, V. V. Shvartsman, M. Ziolkowski, M. Vogt, J. Strempfer, T. Łukasievic, U. Pietsch, J. Dec. Phys. Rev. Lett 114, 097601, (2015).

Keywords: ferroelectrics, time-resolved X-ray diffraction electromechanical coupling,

\section{MS22-02 Structural flexibility in prototypical zeolitic imidazolate frameworks} Michael T. Wharmby ${ }^{1}$, Sebastian Henke $^{2}$, Thomas D. Bennett
,
Caroline Mellot-Draznieks ${ }^{3}$, Yuanzheng Yue Y Anthony K. $^{4}$ Cheetham

1. Diamond Light Source Ltd., UK

2. Department of Materials Science \& Metallurgy, University of Cambridge, UK

3. CNRS UMR 8229, Sorbonne Universités, UPMC Univ Paris 06, Collège de France, France

4. Section of Chemistry, Aalborg University, Denmark

email: michael.wharmby@diamond.ac.uk

In recent years, it has become apparent that certain Metal-Organic Framework (MOF) compounds demonstrate remarkable structural flexibility. Understanding this behaviour is crucial to interpreting adsorption in porous compounds. MIL-53 provides the prototypical example, undergoing an anisotropic 'breathing' transition on exchange of adsorbate molecules, by cooling or by application of mechanical pressure. ${ }^{1}$ Phase transitions also influence the gas adsorption behaviour of Zeolitic Imidazolate Frameworks (ZIFs), a significant subclass of MOFs. ZIF-8 demonstrates a gate-opening transition on adsorption of gas molecules and application of mechanical pressure, ${ }^{2,3}$ which is achieved by a rotation of the methylimidazolate linker units to open the windows of the sodalite cages. ZIF-7, also bearing a sodalite framework, demonstrates a slightly different gate-opening transition, achieved by a concomitant rotation of the benzimidazolate linkers and a distortion of the structure. ${ }^{4}$

We report new results in the understanding of the nature of the phase transitions in $\mathrm{ZIF}-7$ and -8 and most significantly, the first example of extreme isotropic flexibility in ZIF compounds. 5 Desolvated ZIF-4(Zn) undergoes an isotropic phase transition on cooling from $298 \mathrm{~K}$ to $80 \mathrm{~K}$, leading to a $23 \%$ reduction in pore volume and a closure of the pore space. This porous to non-porous phase transition was investigated by in situ synchrotron powder X-ray diffraction, which allowed the mechanism to be determined and also to confirm its discontinuous nature. The new low temperature structure is marginally less dense than that of the densest known ZIF phase, ZIF-zni. By combining structural results with DSC measurements and DFT calculations, the driving force for this new and unexpected transition was established. Using these results, it is now possible to understand the gas adsorption behaviour of this important framework compound.

\section{References}

1 Serre et al., Adv. Mater., 2007, 19, 2246-2251.

2 Moggach et al., Angew. Chem. Int. Ed., 2009, 48, 7087-7089.

3 Fairen-Jimenez et al., J. Am. Chem. Soc., 2011, 133, 8900-8902.

4 Zhao et al., Chem. Mater., 2014, 26, 1767-1769.

5 Wharmby et al., Angew. Chem. Int. Ed., 2015, (accepted). 

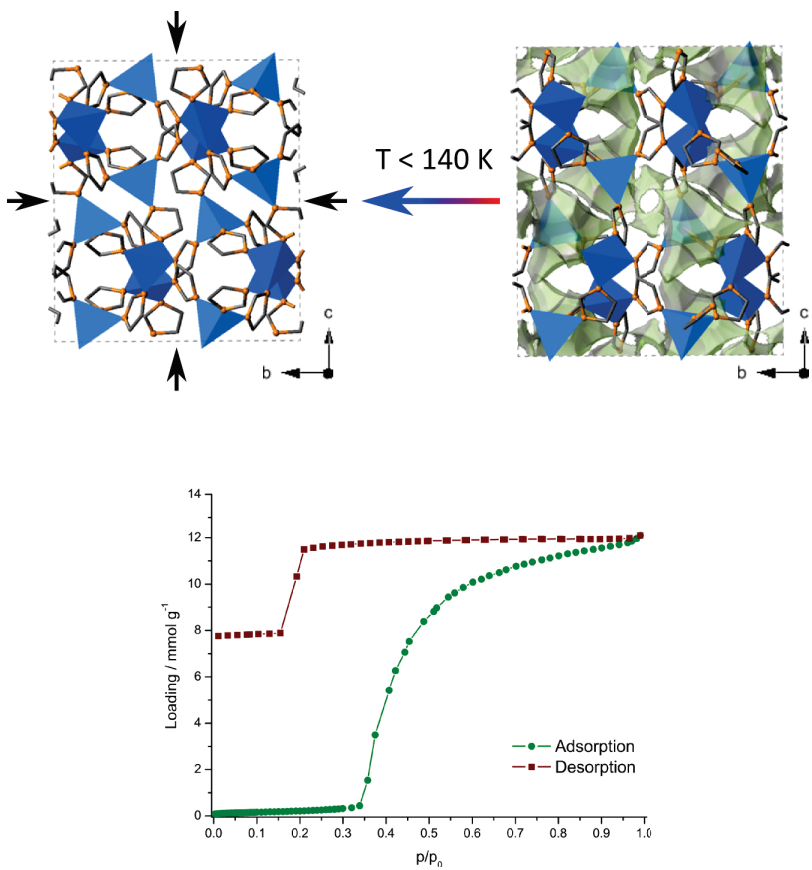

Figure 1. Top: Structural transition at $140 \mathrm{~K}$ in desolvated ZIF-4(Zn), showing the closure of the pore space (highlighted in green). $\mathrm{Zn}$ - blue; C - grey; $\mathrm{N}$ - orange. Bottom: $\mathrm{N}$ adsorption isotherm at $77 \mathrm{~K}$ for $\mathrm{ZIF}-4(\mathrm{Zn})$. Large hysteresis is indicative of structural changes on adsorption.

Keywords: Porous/non-porous phase transition, zeolitic imidazolate framework, ZIF-4, ZIF-7, ZIF-8, low temperature, structure determination from powder data, breathing, gas adsorption, DFT

\section{MS22-O3 Crystallographic features of the dehydration of samarium and yttrium oxalate decahydrates}

Alexander Matvienko ${ }^{1,2}$, Daniel Maslennikov ${ }^{3}$, Pavel Gribov ${ }^{2}$ Stanislav Chizhik ${ }^{1,2}$, Anatoly Sidelnikov ${ }^{1}$, Boris Zakharov ${ }^{1,2}$

1. Institute of Solid State Chemistry and Mechanochemistry, Kutateladze, 18, 630128, Novosibirsk, Russia

2. Novosibirsk State University, Pirogova, 2, 630090, Novosibirsk, Russia.

3. Université Montpellier 2, 34095, Montpellier cedex 5, France

email: matvienko67@gmail.com

The formation of solid product with new crystal structure usually occurs during solid state chemical reaction. The effects of this structural transformation on the morphology and kinetics of the reaction depend on the crystallographic features of a structural transformation. Hence, the ability to design and control the reaction product will depend on the ability to understand, and more importantly, to predict the crystallographic characteristics of a structural transformation. The analysis of the current knowledge and understanding of the crystallographic features of structural transformations is carried out in this work. Structural transformations during phase transformations are divided into two broad classes - the 'reconstructive' transformations and the 'displacive' transformations, exemplified by martensitic or shear transformations. This distinction is largely based on the mechanism of formation (nucleation and growth) of the product phase. The reconstructive transformations are thermally activated, involve migration of incoherent interface. The displacive transformations are associated with a glissile, coherent or partly coherent interface that migrates in a conservative fashion. There is always a correspondence between the matrix and product phase, together with a macroscopic change of shape of the transformed volume. We believe that this classification is valid for the structural transformation during solid state reactions. The comprehensive study of the structural and morphological changes during the dehydration reaction of samarium and yttrium oxalate decahydrates was carried out in this work. X-ray diffraction, TG-DTA methods, optical microscopy, TEM, SEM, Raman spectroscopy and $\mathrm{N}_{2}$ sorption isotherm techniques were used. Basing on the analysis of structures, the orientation relations and shape deformations of crystals the mechanisms of the structural transformation were proposed. These transitions are similar to the displacive or martensitic transformations (Figure). However, the chemical reaction causes the transformation in this case. We used the crystallographic theories of martensitic transformation for the analysis of reaction-related deformation. Detailed analysis of atomic displacements was carried out and strain ellipsoids were obtained for each case. The reaction-related deformation determines the character of the fracture during the reaction. 